\title{
Impact of delisting OTC medicines from reimbursement list
}

\author{
Petra Chytilová*, Robin Šebesta \\ From 3rd International PPRI Conference 2015: Pharmaceutical Pricing and Reimbursement Policies: Challenges \\ Beyond the Financial Crisis \\ Vienna, Austria. 12-13 October 2015
}

\section{Background}

This analysis addresses the impact of policy change when over-the-counter medicines (OTCs) were excluded from the reimbursement list by law since July 1, 2012 in the Czech Republic. Selected OTCs remained reimbursed with the approval of insurance funds, usually under certain conditions of reimbursement (for specific indications or patient groups).

\section{Objectives}

This analysis aims at determining how many OTCs were reimbursed before the exclusion and how many of them and under what conditions of reimbursement remained to be reimbursed. The second objective of the analysis is to estimate the financial effect of exclusion on the payers and patients. The third objective is to describe what type of OTCs are reimbursed and under what conditions of reimbursement, as well as to compare annual costs of reimbursed OTCs versus annual costs of prescription-only medicines (POM).

\section{Methods}

SIDC's list of reimbursed medicines valid as of 1 June 2012 was compared with the list of reimbursed medicines valid as of 1 July 2012 to identify OTCs excluded from reimbursement. The financial impact of delisting OTCs was calculated based on consumption data and reimbursement price from the payers. The current reimbursement list (May 2015) was searched to identify the current spectrum of reimbursed OTCs.

\section{Results}

There were 238 reimbursed OTCs in June 2012 and 14 OTCs remained reimbursed in July 2012, based on registration number. The reimbursement is limited by conditions of reimbursement for most reimbursed OTCs. The delisted OTCs saved payers approximately 21.6 mil EUR (1 EUR $=27.624 \mathrm{CZK})$ in the first year after the delisting. The average price of not excluded OTCs has grown to $12.54 \%$ and the average price of excluded OTCs has grown to $10.70 \%$ since January 2012. The poster will show the analysis of consumption of OTCs in detail.

\section{Conclusions}

Almost $95 \%$ of previously reimbursed OTC medicines were excluded from the reimbursement system in 2012. OTC medicines that remain in the reimbursement system are reimbursed, for example, for patients with cystic fibrosis, diagnosed Sjogren's syndrome (dry eyes), chronic pancreatitis. The payers saved 21.6 mil. EUR due to the delisting. The delisting did not have a major impact on the price of excluded OTCs.

Published: 5 October 2015

doi:10.1186/2052-3211-8-S1-P4

Cite this article as: Chytilová and Šebesta: Impact of delisting OTC

medicines from reimbursement list. Journal of Pharmaceutical Policy and Practice 2015 8(Suppl 1):P4.

\footnotetext{
* Correspondence: petra.chytilova@sukl.cz

State Institute for Drug Control (SIDC), Prague, 100 41, Czech Republic
} 